(Supporting Information)

\title{
Synthesis of a Vanadium(III) Tris(arylthiolato) Complex and Its Reactions with Azide and Azo Compounds; Formation of a Sulfenamide Complex via Cleavage of an Azo $\mathrm{N}=\mathrm{N}$ Bond
}

Takashi Komuro, Tsukasa Matsuo, Hiroyuki Kawaguchi* and Kazuyuki Tatsumi

Institute for Molecular Science, Myodaiji, Okazaki 444-8787, Japan

(submitted to Inorg. Chem.)

\section{Experimental Procedures}

General Procedures. All manipulations were performed under an inert atmosphere of argon using standard Schlenk-line techniques. All dried solvents and chemicals commercially available were used as received without further purification. Deuterated toluene (toluene- $d_{8}$ ) and benzene (benzene- $d_{6}$ ) were dried and degassed over a potassium mirror in vacuo prior to use. Elemental analyses $(\mathrm{C}, \mathrm{H}$, and $\mathrm{N})$ were carried out on a YANACO CHN Corder MT-6 element analyzer. The powder samples were evacuated prior to use. ${ }^{1} \mathrm{H},{ }^{13} \mathrm{C}$ and ${ }^{51} \mathrm{~V}$ NMR spectra were recorded on JEOL Lambda-500 spectrometer. Chemical shifts (in ppm) for ${ }^{1} \mathrm{H}$ NMR spectra were referenced to residual protic solvents peaks. Solution magnetic susceptibilities were determined by the Evans' method and corrected for underlying diamagnetism.

Synthesis of $\mathrm{V}(\mathrm{SAr})_{3}(\mathrm{THF})$ (1) $\left[\mathrm{SAr}=\mathrm{SC}_{6} \mathrm{H}_{3}-2,6-\left(\mathrm{SiMe}_{3}\right)_{2}\right]$. A solution of LiSAr, prepared by reaction of ArSH $(0.81 \mathrm{~g}, 3.2 \mathrm{mmol})$ with BuLi $(2.0 \mathrm{~mL}$ of a $1.58 \mathrm{M}$ solution in hexane, $3.2 \mathrm{mmol})$ in $\mathrm{Et}_{2} \mathrm{O}(20 \mathrm{~mL})$, was added to $\left[\mathrm{VCl}_{3}(\mathrm{THF})_{3}\right](0.39 \mathrm{~g}, 1.0$ 
$\mathrm{mmol})$ in $\mathrm{Et}_{2} \mathrm{O}(5 \mathrm{~mL})$ at $0{ }^{\circ} \mathrm{C}$. After stirring for $5 \mathrm{~min}$ at room temperature, the solution was evaporated to dryness. The residue was dissolved in toluene (20 mL) and then stirred at $60{ }^{\circ} \mathrm{C}$ for $2 \mathrm{~h}$, during which time a color of the solution changed from dark yellow to dark orange. After the solvent was removed in vacuo, the residue was extracted with HMDSO $(15 \mathrm{~mL})$. The extract was concentrated and cooled, yielding $1 \bullet 0.5 \mathrm{HMDSO}$ as dark-orange crystals $(0.81 \mathrm{~g}, 80 \%)$. Compound 1 was crystallized as the HMDSO solvate; elemental analysis (\%) calcd for $\mathrm{C}_{40} \mathrm{H}_{71} \mathrm{OS}_{3} \mathrm{Si}_{6} \mathrm{~V} \bullet 0.5\left(\mathrm{Me}_{3} \mathrm{Si}\right)_{2} \mathrm{O}$ : C 53.53, H 8.36, S 9.97; found: C 53.59, H 8.47, S 9.96; ${ }^{1} \mathrm{H}$ NMR $\left(\mathrm{C}_{6} \mathrm{D}_{6}, 499.10 \mathrm{MHz}\right): \delta=20.6$ (br, $\left.\Delta v_{1 / 2}=260 \mathrm{~Hz}\right)$, $9.8\left(\mathrm{br}, \Delta v_{1 / 2}=220 \mathrm{~Hz}\right), 5.3\left(\mathrm{br}, \Delta v_{1 / 2}=530 \mathrm{~Hz}\right), 0.11\left(\mathrm{~s} ;\left(\operatorname{Si} M e_{3}\right)_{2} \mathrm{O}\right),-4.0 \mathrm{ppm}\left(\mathrm{br}, \Delta v_{1 / 2}=\right.$ $2700 \mathrm{~Hz})$.

Synthesis of V(bit-SAr)(SAr)(TMEDA) (2). A mixture of 1 (0.26 g, 0.27 mmol) and TMEDA ( $50 \mu \mathrm{L}, 0.33 \mathrm{mmol})$ was stirred for $12 \mathrm{~h}$ at room temperature. The resulting dark brown solution was evaporated to dryness, and the residue was triturated with hexane $(5 \mathrm{~mL})$ at $-30{ }^{\circ} \mathrm{C}$, leaving a brick red powder of $2(0.14 \mathrm{~g}, 76 \%)$; elemental analysis (\%) calcd for $\mathrm{C}_{30} \mathrm{H}_{57} \mathrm{~N}_{2} \mathrm{~S}_{2} \mathrm{Si}_{4} \mathrm{~V}$ : C 53.52, H 8.53, N 4.16, S 9.53; found: C 52.93, H 8.40, N 3.75, S 9.30; ${ }^{1} \mathrm{H}$ NMR $\left(\mathrm{C}_{6} \mathrm{D}_{6}, 500 \mathrm{MHz}\right): \delta=15.9\left(\mathrm{br}, \Delta v_{1 / 2}=56 \mathrm{~Hz}\right), 15.6\left(\mathrm{br}, \Delta v_{1 / 2}=43 \mathrm{~Hz}\right)$, $12.3\left(\mathrm{br}, \Delta v_{1 / 2}=110 \mathrm{~Hz}\right), 9.6\left(\mathrm{br}, \Delta v_{1 / 2}=31 \mathrm{~Hz}\right), 7.8\left(\mathrm{br}, \Delta v_{1 / 2}=85 \mathrm{~Hz}\right), 0.9\left(\mathrm{br}, \Delta v_{1 / 2}=180\right.$ $\mathrm{Hz}), 0.3\left(\mathrm{br}, \Delta v_{1 / 2}=68 \mathrm{~Hz}\right),-3.8 \mathrm{ppm}\left(\mathrm{br}, \Delta v_{1 / 2}=260 \mathrm{~Hz}\right)$.

Synthesis of $\mathbf{V}(\mathbf{S A r})_{3}(\mathbf{N A d})(3)$. A toluene solution (4 mL) of 1-adamantyl azide $\left(\right.$ Ad- $\left.\mathrm{N}_{3} ; 0.036 \mathrm{~g}, 0.20 \mathrm{mmol}\right)$ was added to $1(0.19 \mathrm{~g}, 0.20 \mathrm{mmol})$ in toluene $(5 \mathrm{~mL})$ at room temperature. Evolution of gas immediately occurred, and then the mixture was stirred for 13 h. Volatiles were removed under vacuum. The residue was triturated with HMDSO $(5 \mathrm{~mL})$ at $-30{ }^{\circ} \mathrm{C}$ to remove an oily material, leaving an maroon powder of $\mathbf{3}(0.14 \mathrm{~g}, 73 \%)$; 
elemental analysis (\%) calcd for $\mathrm{C}_{46} \mathrm{H}_{78} \mathrm{NS}_{3} \mathrm{Si}_{6} \mathrm{~V}$ : C 57.51, H 8.18, N 1.46, S 10.01; found: C 57.01, H 8.12, N 1.39, S 9.54; ${ }^{1} \mathrm{H}$ NMR $\left(\mathrm{C}_{6} \mathrm{D}_{6}, 399.65 \mathrm{MHz}\right): \delta=7.52(\mathrm{~d}, 6 \mathrm{H}, J=7.3$ $\mathrm{Hz} ; m$-ArH), 7.02 (t, $3 \mathrm{H}, J=7.3 \mathrm{~Hz} ; p$-ArH), 2.04-2.14 (m, $6 \mathrm{H} ; \mathrm{NC}_{10} \mathrm{H}_{15}$ ), 1.90 (br, $3 \mathrm{H}$; $\left.\mathrm{NC}_{10} \mathrm{H}_{15}\right), 1.34-1.53\left(\mathrm{~m}, 6 \mathrm{H} ; \mathrm{NC}_{10} \mathrm{H}_{15}\right), 0.47 \mathrm{ppm}\left(\mathrm{s}, 54 \mathrm{H} ; \mathrm{SiMe}_{3}\right) ;{ }^{13} \mathrm{C} \mathrm{NMR}\left(\mathrm{C}_{6} \mathrm{D}_{6}, 100.40\right.$ MHz, rt): $\delta=161.9,142.9,136.2,126.2$ (Ar), 92.6, 44.2, 35.8, 30.1 (Ad), 2.4 (SiMe) ppm; ${ }^{51} \mathrm{~V}$ NMR $\left(\mathrm{C}_{6} \mathrm{D}_{6}, 131.43 \mathrm{MHz}, \mathrm{rt}\right): \delta=720\left(\Delta v_{1 / 2}=980 \mathrm{~Hz}\right) \mathrm{ppm}$.

Synthesis of V(NPh)(bit-PhNSAr)(SAr) (4). A mixture of 1 (0.49 g, $0.51 \mathrm{mmol})$ and azobenzene $(0.092 \mathrm{~g}, 0.50 \mathrm{mmol})$ in toluene $(15 \mathrm{~mL})$ was refluxed for $19 \mathrm{~h}$. After removal of volatiles in vacuo, the residue was dissolved in HMDSO $(7.5 \mathrm{~mL})$ to remove free ArSH. Cooling to $-30{ }^{\circ} \mathrm{C}$ yielded the crude product as a brownish orange powder $(0.23 \mathrm{~g}, 61 \%)$. Recrystallization from $\mathrm{Et}_{2} \mathrm{O} / \mathrm{HMDSO}$ afforded analytically pure 4 as orange crystals $(0.14 \mathrm{~g}, 37 \%)$; elemental analysis (\%) calcd for $\mathrm{C}_{36} \mathrm{H}_{51} \mathrm{~N}_{2} \mathrm{~S}_{2} \mathrm{Si}_{4} \mathrm{~V}: \mathrm{C} 58.49, \mathrm{H}$ 6.95, N 3.79, S 8.68; found: C 58.10, H 6.96, N 3.76, S 8.33; ${ }^{1} \mathrm{H}$ NMR $\left(\mathrm{C}_{6} \mathrm{D}_{6}, 500 \mathrm{MHz}, \mathrm{rt}\right)$ : $\delta=7.36-7.67(\mathrm{~m}, 6 \mathrm{H} ; \mathrm{ArH}), 6.56-7.20(\mathrm{~m}, 10 \mathrm{H} ; \mathrm{ArH}), 3.81\left(\mathrm{~d}, 1 \mathrm{H}, J=10 \mathrm{~Hz} ; \mathrm{VCH}_{2}\right)$, $2.22\left(\mathrm{~d}, 1 \mathrm{H}, J=10 \mathrm{~Hz} ; \mathrm{VCH}_{2}\right), 0.65$ (s, $\left.9 \mathrm{H} ; \mathrm{SiMe}_{3}\right), 0.50$ (s, $\left.9 \mathrm{H}, \mathrm{SiMe}_{3}\right), 0.26$ (s, $9 \mathrm{H}$, $\mathrm{SiMe}_{3}$ ), 0.19 (s, $\left.3 \mathrm{H} ; \mathrm{SiMe} \mathrm{CH}_{2}\right), 0.18 \mathrm{ppm}\left(\mathrm{s}, 3 \mathrm{H}\right.$; $\left.\mathrm{SiMe}{ }_{2} \mathrm{CH}_{2}\right) ;{ }^{13} \mathrm{C} \mathrm{NMR}\left(\mathrm{C}_{6} \mathrm{D}_{6}, 100.40\right.$ $\mathrm{MHz}, \mathrm{rt}): \delta=162.5,160.5,153.6,147.2,143.0,142.1,141.9,138.4,136.9,136.6,136.0$, 128.6, 126.4, 126.3, 125.3, 124.6, 119.5 (Ar), 72.3 (br, $\left.\Delta v_{1 / 2}=360 \mathrm{~Hz} ; \mathrm{V}-C\right), 4.4,1.9,1.7$, $1.6(\mathrm{SiMe}) \mathrm{ppm} ;{ }^{51} \mathrm{~V} \mathrm{NMR}\left(\mathrm{C}_{6} \mathrm{D}_{6}, 131.43 \mathrm{MHz}, \mathrm{rt}\right): \delta=224\left(\Delta v_{1 / 2}=570 \mathrm{~Hz}\right) \mathrm{ppm}$.

X-ray Crystallography. Single crystals of $\mathbf{1}$ were obtained from the HMDSO solution, while those of $\mathbf{2}$ and $\mathbf{3}$ were obtained by the slow diffusion of hexane into toluene a solution of the complex. A slow diffusion of HDMSO into an $\mathrm{Et}_{2} \mathrm{O}$ solution of 4 afforded single crystals. Diffraction data of $\mathbf{1 - 4}$ were collected at $-100{ }^{\circ} \mathrm{C}$ on a Rigaku 
Mercury CCD diffractometer or a Rigaku Saturn CCD diffractometer $\left(\mathrm{Mo}_{\mathrm{K} \alpha}\right.$ radiation). The structures were solved by the Patterson method and refined by full-matrix least-squares on $F^{2}$ by using the CrystalStructure software package. All non-hydrogen atoms except for the disordered atoms were refined anisotropically, and hydrogen atoms were located at calculated positions. In the case of $\mathbf{1}$, atoms of the crystal solvent HDMSO were disordered with the occupancy factors of 50:50, and three $\mathrm{C}-\mathrm{H}$ protons of the methyl group $\mathrm{C}(7)$ have been found in the final difference Fourier map. One trimethylsilyl group Si(4) of the arylthiolate ligand in 2 was disordered, resulting in the occupancy factors of 50:50.

Crystal data for $1 \bullet 0.5\left(\mathrm{Me}_{3} \mathrm{Si}\right)_{2} \mathrm{O}: \mathrm{C}_{43} \mathrm{H}_{80} \mathrm{O}_{1.5} \mathrm{Si}_{7} \mathrm{~S}_{3} \mathrm{~V}\left(M_{\mathrm{r}}=964.82\right) ; P-1$ (no. 2), $a=$

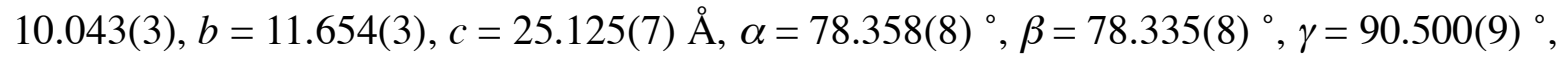
$V=2816.8(14) \AA^{3}, d_{\text {calc }}=1.137 \mathrm{~g} / \mathrm{cm}^{3}, Z=2, \mu=4.65 \mathrm{~cm}^{-1}, 22051$ measured, 12285 unique, 577 variables, $\mathrm{GOF}=1.001, R 1=0.052(I>2 \sigma(I)), w R_{2}=0.146$ (all unique reflections), largest difference peak and hole $=0.82$ and $-0.66 \mathrm{e} \AA^{-3}$, respectively.

For 2: $\mathrm{C}_{30} \mathrm{H}_{57} \mathrm{~N}_{2} \mathrm{Si}_{4} \mathrm{~S}_{2} \mathrm{~V}\left(M_{\mathrm{r}}=673.20\right) ; P 2_{1} / c$ (no. 14), $a=9.338(4), b=19.654(7), c=$ 21.400(9) $\AA, \beta=106.141(6)^{\circ}, V=3772.7(26) \AA^{3}, d_{\text {calc }}=1.185 \mathrm{~g} / \mathrm{cm}^{3}, Z=4, \mu=5.22 \mathrm{~cm}^{-1}$, 60464 measured, 8863 unique, 392 variables, $\mathrm{GOF}=1.001, R 1=0.082, w R_{2}=0.245$, largest difference peak and hole $=1.31$ and $-1.08 \mathrm{e} \AA^{-3}$, respectively.

For 3: $\mathrm{C}_{46} \mathrm{H}_{78} \mathrm{NSi}_{6} \mathrm{~S}_{3} \mathrm{~V}\left(M_{\mathrm{r}}=960.76\right) ; P 2_{1} / c$ (no. 14), $a=12.295(3), b=16.438(4), c=$ 27.386(7) $\AA, \beta=99.814(3)^{\circ}, V=5454.0(25) \AA^{3}, d_{\text {calc }}=1.170 \mathrm{~g} / \mathrm{cm}^{3}, Z=4, \mu=4.58 \mathrm{~cm}^{-1}$, 87630 measured, 12920 unique, 592 variables, $\mathrm{GOF}=1.001, R 1=0.055, w R_{2}=0.154$, largest difference peak and hole $=0.94$ and -0.64 e $\AA^{-3}$, respectively.

For 4: $\mathrm{C}_{36} \mathrm{H}_{51} \mathrm{~N}_{2} \mathrm{Si}_{4} \mathrm{~S}_{2} \mathrm{~V}\left(M_{\mathrm{r}}=739.22\right) ; P 2_{1} / c$ (no. 14), $a=20.069(5), b=9.974(2), c=$ 21.713(5) $\AA, \beta=111.376(4)^{\circ}, V=4047.1(16) \AA^{3}, d_{\text {calc }}=1.213 \mathrm{~g} / \mathrm{cm}^{3}, Z=4, \mu=4.93 \mathrm{~cm}^{-1}$, 60952 measured, 9545 unique, 457 variables, $\mathrm{GOF}=1.014, R 1=0.053, w R_{2}=0.107$, largest difference peak and hole $=1.28$ and $-0.85 \mathrm{e}^{-3}$, respectively. 
Figure S1. Structure of $\mathrm{V}(\mathrm{SAr})_{3}(\mathrm{NAd})(\mathbf{3})$.

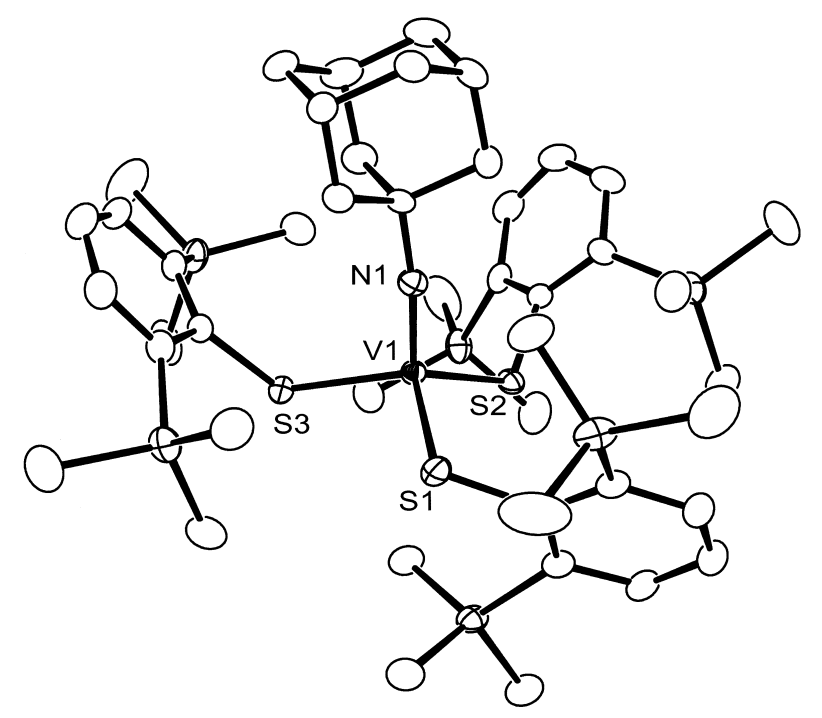

Table S1. Selected Bond Dsitances $(\AA)$ and Angles (deg) of V(SAr) $)_{3}(\mathrm{NAd})(3)$.

\begin{tabular}{llll}
\hline $\mathrm{V}(1)-\mathrm{S}(1)$ & $2.3191(7)$ & $\mathrm{S}(1)-\mathrm{V}(1)-\mathrm{S}(2)$ & $108.97(3)$ \\
$\mathrm{V}(1)-\mathrm{S}(2)$ & $2.2460(7)$ & $\mathrm{S}(1)-\mathrm{V}(1)-\mathrm{S}(3)$ & $105.14(3)$ \\
$\mathrm{V}(1)-\mathrm{S}(3)$ & $2.2658(8)$ & $\mathrm{S}(1)-\mathrm{V}(1)-\mathrm{N}(1)$ & $115.89(8)$ \\
$\mathrm{V}(1)-\mathrm{N}(1)$ & $1.639(2)$ & $\mathrm{S}(2)-\mathrm{V}(1)-\mathrm{S}(3)$ & $115.09(3)$ \\
& & $\mathrm{S}(2)-\mathrm{V}(1)-\mathrm{N}(1)$ & $108.18(8)$ \\
& & $\mathrm{S}(3)-\mathrm{V}(1)-\mathrm{N}(1)$ & $103.73(9)$ \\
& & & \\
\hline
\end{tabular}

